\title{
Genetic variants of IncRNA HOTAIR contribute to the risk of osteosarcoma
}

\author{
Quan Zhou ${ }^{1, *}$, Fengli Chen ${ }^{2, *}$, Zhongting Fei $^{3}$, Jiali Zhao ${ }^{1}$, Yong Liang ${ }^{4}$, Wei Pan ${ }^{1}$, \\ Xingxiang Liu $^{3}$ and Donghui Zheng ${ }^{4}$ \\ ${ }^{1}$ Department of Orthopaedics, Huai'an Hospital Affiliated of Xuzhou Medical College and Huai'an Second Hospital, Huai'an, \\ Jiangsu, China \\ ${ }^{2}$ Department of Central Laboratory, Huai'an First People's Hospital, Nanjing Medical University, Huai'an, Jiangsu, China \\ ${ }^{3}$ Department of Clinical Laboratory, Huai'an 4th people's Hospital, Huai'an, Jiangsu, China \\ ${ }^{4}$ Department of Central Laboratory and Department of Nephrology, Huai'an Hospital Affiliated of Xuzhou Medical College and \\ Huai'an Second Hospital, Huai'an, Jiangsu, China \\ * These authors have contributed equally to this work \\ Correspondence to: Donghui Zheng, email: zhengdonghuijs@163.com
}

Xingxiang Liv, email: Xingxiang_Liv@163.com

Keywords: osteosarcoma, susceptibility, variant, IncRNA, HOTAIR

Received: October 07, $2015 \quad$ Accepted: January 24, 2016

Published: March 07, 2016

\section{ABSTRACT}

Osteosarcoma (OS) is the most common primary malignant bone tumor in adolescents and young adults. However, the essential mechanisms underlying osteosarcomagenesis remain obscure. The HOTAIR, a well-known long noncoding RNA (IncRNA), is involved in pathogenesis and progress of multiple tumors. To reveal the potential role of IncRNA HOTAIR in OS carcinogenesis, we conducted a two-stage, case-control study among Chinese population with 900 OS cases and 900 controls to evaluated associations of its genetic variants with OS risk. We found that $\mathrm{C}$ allele of rs7958904 was associated with a significantly decreased OS risk when compared with G allele (OR: $0.77 ; 95 \% \mathrm{CI}$ : $0.67-0.90 ; P=6.77 \times 10-4$ ). Functional analyses on HOTAIR Expression showed that the expression level of HOTAIR in OS tissues was significantly higher than that in corresponding normal tissues, and subjects with the rs7958904 CC genotype had significantly lower HOTAIR RNA levels than those of other genotypes. This should be the first study to examine the association between HOTAIR variants and OS risk.

\section{INTRODUCTION}

Osteosarcoma (OS), which derives from primitive bone-forming mesenchymal cells, is the most common primary bone malignancy and the eighth-most common form of childhood cancer, comprising $2.4 \%$ of all malignancies in pediatric patients, and about $20 \%$ of all primary bone cancers [1-5]. According to the Surveillance, Epidemiology, and End Results (SEER) program, approximately 7,104 cases of malignant primary OS patients were identified during 1999-2008 in United States, of which 5,379 were appendicular and 1,725 were axial [3]. For many years, accumulated evidences suggested that multiple genetic and environmental factors play pivotal roles in the pathogenesis of OS $[6,7]$. However, the essential mechanisms underlying osteosarcomagenesis and progression continue to be obscure [8-10].

Recently, long non-coding RNAs (lncRNAs) have been identified for their wide range of biological regulatory functions in the carcinogenesis and progression of many cancers [11-14]. Loss of HOTAIR, a lncRNA in the mammalian HOXC locus that binds to and targets the PRC2 complex to the HOXD locus, can inhibit cancer invasiveness, particularly in cells that possess excessive PRC2 activity [15]. It has been widely explored for its genetic variants, expression level and carcinogenesis, tumor development and progression [16-19]. Previously, studies have investigated the association between HOTAIR variants and several cancers, such as breast cancer, gastric cancer, colorectal cancer, esophageal squamous cell carcinoma, and gastric cardia adenocarcinoma $[17,20-$ 25]. However, little is known about the role of these gene 
polymorphisms in the carcinogenesis of OS. Thus, to clarify this association, we analyzed the role of HOTAIR variants in OS Development in a two-stage case-control study among a Chinese population.

\section{RESULTS}

\section{Characteristics of the study population}

Table 1 presents the distribution of selected characteristics among OS cases and controls. In current study, a total of 900 OS cases and 900 controls were recruited in two stages, and there were no significant differences between OS cases and healthy controls for each stage regarding to age and gender (all $P>0.05$ ), which indicates that the frequency matching was adequate. The tumors were mainly located at extremities, and half of the cases were metastatic OS. About 39.8\% of cases' enneking stage were defined as III in stage 1 , and $40.1 \%$ in stage 2 .

\section{Associations of tagSNPs and OS risk}

In stage 1, the genotypes distributions of 3 SNPs (rs4759314, rs7958904 and rs874945) among the controls were in accordance with Hardy-Weinberg equilibrium (all $P>0.05)$. The genotype distribution of all the tagSNPs and their associations with OS risk in our Stage 1 are shown in Table 2. SNP rs7958904 and rs874945 showed significant associations with OS risk in log-additive model ( $P$ value $=0.023,0.024$ respectively $)$. For rs7958904, $\mathrm{C}$ allele was associated a significantly decreased OS risk when compared with $\mathrm{G}$ allele (OR: 0.79; 95\% CI: 0.65-0.97); while for rs874945, A allele was associated a significantly increased OS risk when compared with G allele (OR: 1.28; 95\% CI: 1.03-1.59). No notable associations between rs4759314 and OS risk in the Stage 1 were observed. Therefore, we validated the effects of rs874945 and rs7958904 in the Stage 2, to further confirm the association observed in the first stage. As shown in Table 2, the association of rs874945 disappeared in the Stage $2\left(P_{\text {trend }}=0.614\right)$. However, the protective effects of rs7958904 still existed in the Stage $2\left(P_{\text {trend }}=0.011\right)$. When combined together, $\mathrm{C}$ allele was significantly associated with a decreased OS risk when compared with G allele (OR: 0.77; 95\% CI: 0.67-0.90; $P=6.77 \times 10^{-4}$ ). The adjusted OR for the carriers with the CG genotype was 0.82 (95\% CI: 0.67-1.00) and for those with the CC genotype was 0.57 (95\% CI: 0.40-0.81) compared with the GG genotype. We also conducted stratified analyses by age, gender, and tumor location (Table 3 ). The results didn't change materially.

\section{Functional relevance of rs7958904 on HOTAIR expression}

To confirm the abnormal expression of HOTAIR in OS patients, we evaluated the HOTAIR levels in 100 paired tissues of OS patients and corresponding normal tissues. As shown in Figure 1, the expression level of HOTAIR in OS tissues was significantly higher than that in corresponding normal tissues $(P=0.008)$. Subjects with the rs7958904 CC genotype had significantly lower HOTAIR RNA levels (mean \pm SD) than those with the GG genotypes in normal and OS tissues $(P<0.01)$.

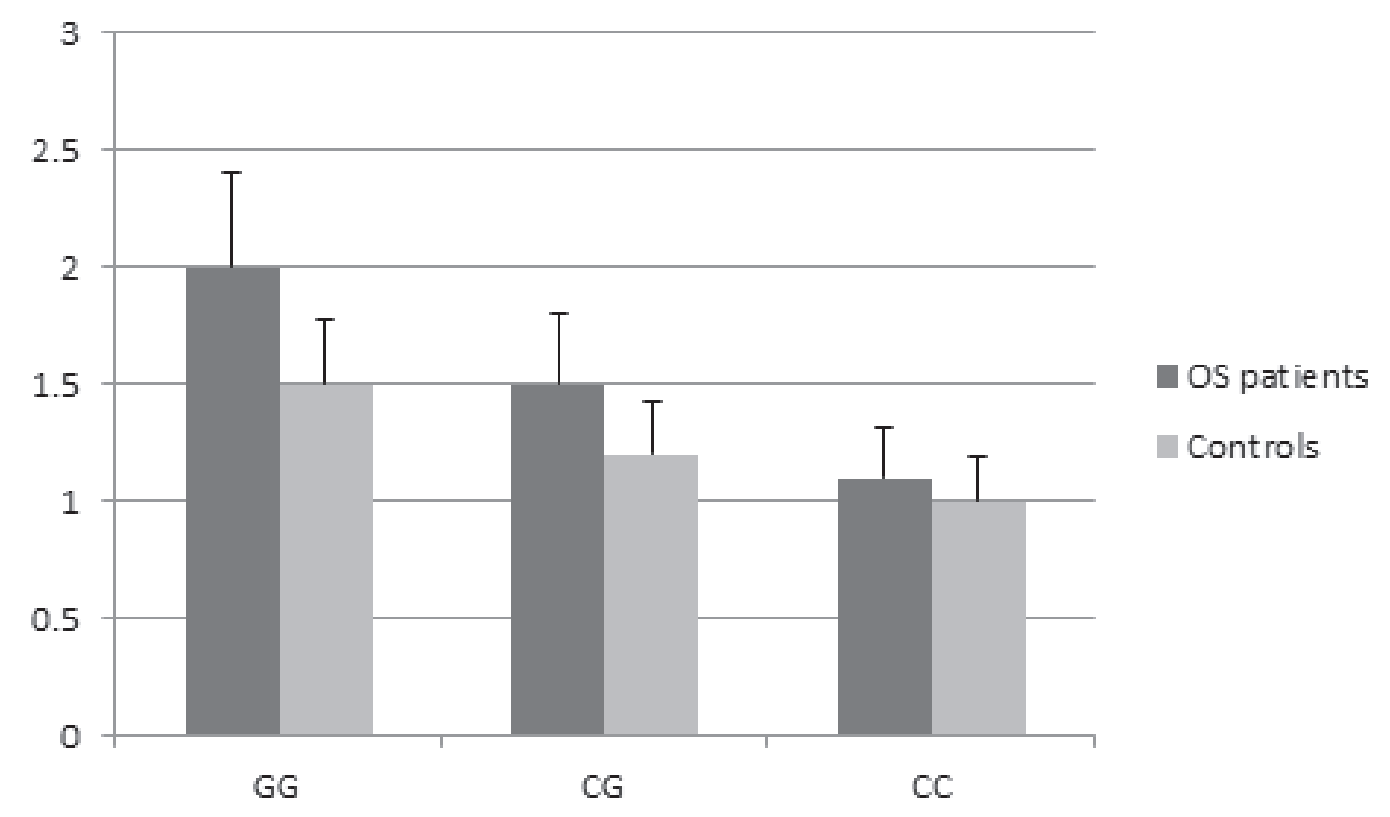

Figure 1: HOTAIR RNA expression (mean \pm SD) in normal and OS tissues grouped by SNP rs7958904 of HOTAIR. 
Table 1: Distribution of selected characteristics among osteosarcoma cases and controls

\begin{tabular}{|c|c|c|c|c|c|c|}
\hline \multirow[b]{2}{*}{ Category } & \multicolumn{3}{|l|}{ Stage 1} & \multicolumn{3}{|l|}{ Stage 2} \\
\hline & Cases $(N=500)$ & Controls $(N=500)$ & $P$ Value & Cases $(N=400)$ & Controls $(N=400)$ & $P$ Value \\
\hline \multicolumn{7}{|l|}{ Age (yr) } \\
\hline Mean \pm SD & $23.5 \pm 8.5$ & $23.3 \pm 7.1$ & 0.686 & $23.7 \pm 5.5$ & $23.2 \pm 5.2$ & 0.187 \\
\hline \multicolumn{7}{|l|}{ Gender } \\
\hline Male & $300(60.0 \%)$ & $295(58.9 \%)$ & 0.747 & $237(59.2 \%)$ & $238(59.5 \%)$ & 0.943 \\
\hline Female & $200(40.0 \%)$ & $205(41.1 \%)$ & & $163(40.8 \%)$ & $162(40.5 \%)$ & \\
\hline \multicolumn{7}{|c|}{ Tumor location } \\
\hline Extremities & $394(78.7 \%)$ & & & $320(80.0 \%)$ & & \\
\hline Other & $106(21.3 \%)$ & & & $80(20.0 \%)$ & & \\
\hline \multicolumn{7}{|l|}{ Metastasis } \\
\hline Yes & $238(47.5 \%)$ & & & $207(51.8 \%)$ & & \\
\hline No & $262(52.5 \%)$ & & & $193(48.2 \%)$ & & \\
\hline \multicolumn{7}{|c|}{ Enneking stage } \\
\hline I-II & $301(60.2 \%)$ & & & $240(59.9 \%)$ & & \\
\hline III & $199(39.8 \%)$ & & & $160(40.1 \%)$ & & \\
\hline
\end{tabular}

Table 2: Genetic variants of HOTAIR and Osteosarcoma risk

\begin{tabular}{|c|c|c|c|c|}
\hline Stage & Genotype & Cases & Controls & Adjusted OR (95\% CI)* \\
\hline \multirow[t]{5}{*}{ rs4759314 } & $\mathrm{AA}$ & 423 & 425 & 1.00 (reference) \\
\hline & $\mathrm{AG}$ & 62 & 64 & $0.97(0.67-1.42)$ \\
\hline & GG & 15 & 11 & $1.37(0.62-3.01)$ \\
\hline & G vs A & & & $1.08(0.79-1.47)$ \\
\hline & $\mathrm{P}$ trend & & & 0.638 \\
\hline \multicolumn{5}{|l|}{ rs874945 } \\
\hline \multirow[t]{5}{*}{ Stage 1} & GG & 310 & 338 & 1.00 (reference) \\
\hline & $\mathrm{AG}$ & 150 & 135 & $1.21(0.92-1.60)$ \\
\hline & $\mathrm{AA}$ & 40 & 27 & $1.62(0.97-2.68)$ \\
\hline & A vs $G$ & & & $1.28(1.03-1.59)$ \\
\hline & $\mathrm{P}$ trend & & & 0.024 \\
\hline \multirow[t]{5}{*}{ Stage 2} & GG & 267 & 270 & 1.00 (reference) \\
\hline & $\mathrm{AG}$ & 106 & 108 & $0.99(0.72-1.36)$ \\
\hline & $\mathrm{AA}$ & 27 & 22 & $1.24(0.69-2.23)$ \\
\hline & A vs $G$ & & & $1.07(0.83-1.36)$ \\
\hline & $\mathrm{P}$ trend & & & 0.614 \\
\hline \multicolumn{5}{|l|}{ rs7958904 } \\
\hline \multirow[t]{5}{*}{ Stage 1} & GG & 295 & 266 & 1.00 (reference) \\
\hline & $\mathrm{CG}$ & 180 & 194 & $0.83(0.64-1.09)$ \\
\hline & $\mathrm{CC}$ & 25 & 40 & $0.56(0.33-0.95)$ \\
\hline & C vs $\mathrm{G}$ & & & $0.79(0.65-0.97)$ \\
\hline & $\mathrm{P}$ trend & & & 0.023 \\
\hline \multirow[t]{5}{*}{ Stage 2} & GG & 229 & 200 & 1.00 (reference) \\
\hline & $\mathrm{CG}$ & 140 & 152 & $0.80(0.60-1.08)$ \\
\hline & $\mathrm{CC}$ & 31 & 48 & $0.56(0.35-0.92)$ \\
\hline & C vs G & & & $0.75(0.60-0.93)$ \\
\hline & $\mathrm{P}$ trend & & & 0.011 \\
\hline \multirow[t]{5}{*}{ Combined results } & GG & 524 & 466 & 1.00 (reference) \\
\hline & $\mathrm{CG}$ & 320 & 346 & $0.82(0.67-1.00)$ \\
\hline & $\mathrm{CC}$ & 56 & 88 & $0.57(0.40-0.81)$ \\
\hline & C vs G & & & $0.77(0.67-0.90)$ \\
\hline & $\mathrm{P}$ trend & & & $6.77 \times 10^{-4}$ \\
\hline
\end{tabular}

* Adjusting for age, and gender 
Table 3: HOTAIR rs7958904 and Osteosarcoma risk stratified by co-variables

\begin{tabular}{|c|c|c|c|c|}
\hline Variables & Categories & \begin{tabular}{|l|} 
Genotype \\
\end{tabular} & Adjusted OR (95\% CI) & P trend \\
\hline \multicolumn{5}{|l|}{ Gender } \\
\hline & Male & GG & 1.00 (reference) & \\
\hline & & $\mathrm{CG}$ & $0.81(0.63-1.03)$ & \\
\hline & & $\mathrm{CC}$ & $0.55(0.39-0.78)$ & \\
\hline & & C vs G & $0.79(0.66-0.94)$ & 0.010 \\
\hline & Female & GG & 1.00 (reference) & \\
\hline & & $\mathrm{CG}$ & $0.84(0.69-1.02)$ & \\
\hline & & $\mathrm{CC}$ & $0.58(0.39-0.87)$ & \\
\hline & & C vs G & $0.74(0.57-0.95)$ & 0.019 \\
\hline \multirow[t]{8}{*}{ Tumor location } & Extremities & GG & 1.00 (reference) & \\
\hline & & $\mathrm{CG}$ & $0.81(0.66-1.00)$ & \\
\hline & & $\mathrm{CC}$ & $0.56(0.38-0.82)$ & \\
\hline & & C vs G & $0.77(0.66-0.90)$ & 0.001 \\
\hline & Other & GG & 1.00 (reference) & \\
\hline & & $\mathrm{CG}$ & $0.85(0.70-1.03)$ & \\
\hline & & $\mathrm{CC}$ & $0.59(0.37-0.95)$ & \\
\hline & & C vs $\mathrm{G}$ & $0.79(0.63-0.99)$ & 0.041 \\
\hline \multirow[t]{8}{*}{ Age } & $\geq 23.5$ & GG & 1.00 (reference) & \\
\hline & & $\mathrm{CG}$ & $0.84(0.70-1.01)$ & \\
\hline & & $\mathrm{CC}$ & $0.59(0.42-0.82)$ & \\
\hline & & C vs G & $0.79(0.66-0.95)$ & 0.011 \\
\hline & $<23.5$ & GG & 1.00 (reference) & \\
\hline & & $\mathrm{CG}$ & $0.79(0.61-1.03)$ & \\
\hline & & $\mathrm{CC}$ & $0.56(0.36-0.87)$ & \\
\hline & & C vs G & $0.75(0.59-0.95)$ & 0.020 \\
\hline
\end{tabular}

\section{DISCUSSION}

Deeper understanding of lncRNAs and their role in carcinogenesis could possess a large number of potential clues for developing novel therapeutic agents for OS. In this two-stage, case-control study, we examined the relationship between HOTAIR variants and OS risk among Chinese population. We identified that people with $\mathrm{C}$ allele of SNP rs7958904 had a decreased risk of developing OS. SNP rs7958904 also has a genotype-specific effect on lncRNA HOTAIR expression. Our findings support the hypothesis that functional genetic variants influencing IncRNA expression may explain a part of OS genetic basis. To our knowledge, this is the first study to examine the association between HOTAIR variants and OS risk.

lncRNA HOTAIR has been widely explored as a functional lncRNA participating in multiple cancers [26]. In 2007, Rinn et al [27] first characterized one wellplaced lncRNA in HOX clusters: this 2158 nucleotide RNA, termed HOTAIR, was localized to a regulatory boundary in the HOXC cluster and was expressed in both distal and posterior fibroblasts. This means HOTAIR lifts noncoding RNAs to new levels [28]. Since then, HOTAIR was evaluated as an oncogenic factor and could be used as a prognostic biomarker in different cancer type [29]. In a meta-analysis with 748 patients from 8 studies, Cai et al [30] showed that the patients with high HOTAIR expression level had a higher incidence compared with that in patients with low HOTAIR expression level. On the other hand, suppressed expression of HOTAIR inhibits the proliferation and tumorigenesis both in vitro and in vivo [31]. Except of these, HOTAIR was additionally reported to have significant influence on the proliferation, metastasis, EMT, and drug resistance in various human cancers [29]. Conclusively, understanding the biological roles of HOTAIR in different cancer types may help us to recruit this IncRNA as a diagnostic or predictive biomarker.

In current study, we determined $\mathrm{C}$ allele of HOTAIR rs7958904 could decrease the OS risk, as well as the expression level of HOTAIR (OR: $0.77 ; 95 \% \mathrm{CI}$ : 0.67-0.90). These results was consistent with a previous study by Xue et al [18], which identified individuals with rs7958904 CC genotype had a significantly decreased risk of colorectal cancer in both Stage 1 and 2, compared with those carrying GG genotype $(\mathrm{OR}=0.67,95 \% \mathrm{CI}=0.51$ 0.87 in combined stage). Through in silico analysis, the secondary structure of HOTAIR was remarkably changed 
with rs7958904 G/C variant [18]. However, another SNP rs4759314, which was significantly associated with the increased gastric cancer risk by $\mathrm{Du}$ et al [17], was not replicated in current study.

In conclusion, we identified a SNP located in HOTAIR gene (rs7958904) that was significantly associated with decreased risk of OS in our two-stage, case-control study. Furthermore, in vivo studies found HOTAIR was notably up-regulated in OS tissues than in adjacent normal tissues, and subjects with the rs7958904 CC genotype had significantly lower HOTAIR RNA levels than other genotypes. Strength of this study includes large sample size, study design, and homogeneous participants. Larger prospective studies and further studies into the detailed biological mechanisms of HOTAIR SNPs are warranted to confirm our results.

\section{MATERIALS AND METHODS}

\section{Subjects}

Totally included in this study were 500 subjects diagnosed with OS, and 500 healthy controls in stage 1, as well as 400 OS patients and 400 healthy controls in an independent validation stage (stage 2). Pathological diagnosis was made by an experienced pathologist and double-checked by another pathologist. The healthy controls free from any cancer and matched by gender and age, were recruited when they were attending a routine examination. Blood samples $(5 \mathrm{ml})$ were obtained from the subjects who participated in the study. A structured questionnaire was used to elicit detailed information on demographic factors. All specimens were handled and made anonymous according to the ethical and legal standards. This study was approved by the institutional Review Boards. Written informed consents were obtained according to the Declaration of Helsinki from both groups.

\section{SNP selection and genotyping}

Genomic DNA was extracted from peripheral blood mononuclear cells using the QIAamp DNA whole blood kit (QIAGEN Inc., Valencia, CA, USA). The tagSNPs were selected basing on data of Han Chinese population (HCB data) of the HapMap Project (HapMap Rel 27, NCBI B36) covering 10232bp region (6232bp HOTAIR locus and $2 \mathrm{~kb}$ upstream as well as $2 \mathrm{~kb}$ downstream regions of the HOTAIR gene). Three htSNPs (rs4759314, rs7958904 and rs874945) were selected with Haploview version 4.2 software. SNP genotyping was performed by the Sequenom MassARRAY RS1000 while Sequenom Typer 4.0 Software was used to perform data management and analysis. The genotyping analysis was done blind as regards participants. To control the quality, the selected
PCR-amplified DNA samples were examined by DNA sequencing to confirm genotyping results.

\section{Quantitative real-time RT-PCR analyses of HOTAIR}

A total of 100 OS tissues were obtained from pretreatment patients. RNA was extracted from the frozen tumor and corresponding normal tissues by standard methods using TRIzol reagent (Invitrogen, Carlsbad, CA, USA) according to the manufacturer's instructions. The expression of HOTAIR was determined by SYBR Green Assay and the levels was calculated relative to expression of $\beta$-actin by the $2^{-\Delta \mathrm{Ct}}$ method. All assays were conducted by using the ABI 7900 HT system (Applied Biosystems, Foster City, CA, USA). All reactions were performed in triplicate.

\section{Statistical analyses}

Comparisons between groups were made using the $\mathrm{x}^{2}$ test (nominal data) or Student's t test (continuous data). Hard-Weinberg analysis was performed by comparing the observed and expected genotype frequencies of HOTAIR using chi-square test. The odds ratios (OR), with $95 \%$ confidence interval (CI), were calculated by an unconditional logistic regression model. We evaluated the log-additive (which assumes an additive effect of each copy of the minor

allele) inheritance models for each SNP in relationship to OS case status. Statistical analysis was performed using SPSS13.0 software package (SPSS Company, Chicago, IL, USA). $P$ value $<0.05$ was considered statistically significant.

\section{ACKNOWLEDGMENTS}

This research was supported by grants from the scientific research fund of the Bureau of Public Health of Jiangsu province (No H201254) and the Huai'an Technology Support Program (social development) funded projects (No HAS2013046, No HAS2015009-4).

\section{CONFLICTS OF INTEREST}

The authors declare that they have no conflicts of interest.

\section{REFERENCES}

1. Duchman KR, Gao Y and Miller BJ. Prognostic factors for survival in patients with high-grade osteosarcoma using the Surveillance, Epidemiology, and End Results (SEER) Program database. Cancer Epidemiol. 2015; 39:593-599. 
2. Ottaviani G and Jaffe N. The epidemiology of osteosarcoma. Cancer treatment and research. 2009; 152:3-13.

3. Duong LM and Richardson LC. Descriptive epidemiology of malignant primary osteosarcoma using population-based registries, United States, 1999-2008. Journal of registry management. 2013; 40:59-64.

4. Sampo M, Koivikko M, Taskinen M, Kallio P, Kivioja A, Tarkkanen $\mathrm{M}$ and Bohling T. Incidence, epidemiology and treatment results of osteosarcoma in Finland - a nationwide population-based study. Acta Oncol. 2011; 50:1206-1214.

5. Buckley JD, Pendergrass TW, Buckley CM, Pritchard DJ, Nesbit ME, Provisor AJ and Robison LL. Epidemiology of osteosarcoma and Ewing's sarcoma in childhood: a study of 305 cases by the Children's Cancer Group. Cancer. 1998; 83:1440-1448.

6. Bonuccelli G, Avnet S, Grisendi G, Salerno M, Granchi D, Dominici M, Kusuzaki K and Baldini N. Role of mesenchymal stem cells in osteosarcoma and metabolic reprogramming of tumor cells. Oncotarget. 2014; 5:75757588. doi: 10.18632/oncotarget.2243.

7. Mirabello L, Koster R, Moriarity BS, Spector LG, Meltzer PS, Gary J, Machiela MJ, Pankratz N, Panagiotou OA, Largaespada D, Wang Z, Gastier-Foster JM, Gorlick R, Khanna C, de Toledo SR, Petrilli AS, et al. A GenomeWide Scan Identifies Variants in NFIB Associated with Metastasis in Patients with Osteosarcoma. Cancer discovery. 2015; 5:920-931.

8. Lamoureux F, Baud'huin M, Ory B, Guiho R, Zoubeidi A, Gleave M, Heymann D and Redini F. Clusterin inhibition using OGX-011 synergistically enhances zoledronic acid activity in osteosarcoma. Oncotarget. 2014; 5:7805-7819. doi: 10.18632/oncotarget.2308.

9. Tsai HC, Su HL, Huang CY, Fong YC, Hsu CJ and Tang CH. CTGF increases matrix metalloproteinases expression and subsequently promotes tumor metastasis in human osteosarcoma through down-regulating miR519d. Oncotarget. 2014; 5:3800-3812. doi: 10.18632/ oncotarget.1998.

10. Yu L, Liu S, Zhang C, Zhang B, Simoes BM, Eyre R, Liang Y, Yan H, Wu Z, Guo W and Clarke RB. Enrichment of human osteosarcoma stem cells based on hTERT transcriptional activity. Oncotarget. 2013; 4:2326-2338. doi: 10.18632/oncotarget.1554.

11. Li Y, Chen J, Zhang J, Wang Z, Shao T, Jiang C, Xu J and Li X. Construction and analysis of lncRNA-lncRNA synergistic networks to reveal clinically relevant lncRNAs in cancer. Oncotarget. 2015; 6:25003-25016. doi: 10.18632/ oncotarget.1554.

12. Li J, Han L, Roebuck P, Diao L, Liu L, Yuan Y, Weinstein JN and Liang H. TANRIC: An Interactive Open Platform to Explore the Function of IncRNAs in Cancer. Cancer Res. 2015; 75:3728-3737.

13. Prensner JR and Chinnaiyan AM. The emergence of lncRNAs in cancer biology. Cancer discovery. 2011; 1:391-
407.

14. Jin G, Sun J, Isaacs SD, Wiley KE, Kim ST, Chu LW, Zhang Z, Zhao H, Zheng SL, Isaacs WB and Xu J. Human polymorphisms at long non-coding RNAs (lncRNAs) and association with prostate cancer risk. Carcinogenesis. 2011; 32:1655-1659.

15. Gupta RA, Shah N, Wang KC, Kim J, Horlings HM, Wong DJ, Tsai MC, Hung T, Argani P, Rinn JL, Wang Y, Brzoska P, Kong B, Li R, West RB, van de Vijver MJ, et al. Long non-coding RNA HOTAIR reprograms chromatin state to promote cancer metastasis. Nature. 2010; 464:1071-1076.

16. Cai B, Song XQ, Cai JP and Zhang S. HOTAIR: a cancerrelated long non-coding RNA. Neoplasma. 2014; 61:379391.

17. Du M, Wang W, Jin H, Wang Q, Ge Y, Lu J, Ma G, Chu $\mathrm{H}$, Tong N, Zhu H, Wang M, Qiang F and Zhang Z. The association analysis of lncRNA HOTAIR genetic variants and gastric cancer risk in a Chinese population. Oncotarget. 2015; 6:31255-62. doi: 10.18632/oncotarget.5158.

18. Li H, An J, Wu M, Zheng Q, Gui X, Li T, Pu H and Lu D. LncRNA HOTAIR promotes human liver cancer stem cell malignant growth through downregulation of SETD2. Oncotarget. 2015; 6:27847-64. doi: 10.18632/ oncotarget.4443.

19. Li L, Dang Q, Xie H, Yang Z, He D, Liang L, Song W, Yeh $\mathrm{S}$ and Chang $\mathrm{C}$. Infiltrating mast cells enhance prostate cancer invasion via altering LncRNA-HOTAIR/PRC2androgen receptor (AR)-MMP9 signals and increased stem/ progenitor cell population. Oncotarget. 2015; 6:1417914190. doi: 10.18632/oncotarget.3651.

20. Bayram S, Sumbul AT, Batmaci CY and Genc A. Effect of HOTAIR rs920778 polymorphism on breast cancer susceptibility and clinicopathologic features in a Turkish population. Tumour Biol. 2015; 36:3863-3870.

21. Bayram S, Ulger Y, Sumbul AT, Kaya BY, Rencuzogullari A, Genc A, Sevgiler Y, Bozkurt O and Rencuzogullari E. A functional HOTAIR rs920778 polymorphism does not contributes to gastric cancer in a Turkish population: a casecontrol study. Fam Cancer. 2015.

22. Xue Y, Gu D, Ma G, Zhu L, Hua Q, Chu H, Tong N, Chen J, Zhang $Z$ and Wang $M$. Genetic variants in lncRNA HOTAIR are associated with risk of colorectal cancer. Mutagenesis. 2015; 30:303-310.

23. Zhang X, Zhou L, Fu G, Sun F, Shi J, Wei J, Lu C, Zhou C, Yuan Q and Yang M. The identification of an ESCC susceptibility SNP rs920778 that regulates the expression of IncRNA HOTAIR via a novel intronic enhancer. Carcinogenesis. 2014; 35:2062-2067.

24. Pan W, Liu L, Wei J, Ge Y, Zhang J, Chen H, Zhou L, Yuan Q, Zhou C and Yang M. A functional lncRNA HOTAIR genetic variant contributes to gastric cancer susceptibility. Mol Carcinog. 2016; 55:90-6.

25. Guo W, Dong Z, Bai Y, Guo Y, Shen S, Kuang G and Xu J. Associations between polymorphisms of HOTAIR and risk 
of gastric cardia adenocarcinoma in a population of north China. Tumour Biol. 2015; 36:2845-2854.

26. Hajjari $M$ and Salavaty A. HOTAIR: an oncogenic long non-coding RNA in different cancers. Cancer biology \& medicine. 2015; 12:1-9.

27. Rinn JL, Kertesz M, Wang JK, Squazzo SL, Xu X, Brugmann SA, Goodnough LH, Helms JA, Farnham PJ, Segal E and Chang HY. Functional demarcation of active and silent chromatin domains in human HOX loci by noncoding RNAs. Cell. 2007; 129:1311-1323.

28. Woo CJ and Kingston RE. HOTAIR lifts noncoding RNAs to new levels. Cell. 2007; 129:1257-1259.

29. Zhou X, Chen J and Tang W. The molecular mechanism of HOTAIR in tumorigenesis, metastasis, and drug resistance. Acta Biochim Biophys Sin (Shanghai). 2014; 46:1011-
1015.

30. Cai B, Wu Z, Liao $\mathrm{K}$ and Zhang S. Long noncoding RNA HOTAIR can serve as a common molecular marker for lymph node metastasis: a meta-analysis. Tumour Biol. 2014; 35:8445-8450.

31. Huang J, Ke P, Guo L, Wang W, Tan H, Liang Y and Yao S. Lentivirus-mediated RNA interference targeting the long noncoding RNA HOTAIR inhibits proliferation and invasion of endometrial carcinoma cells in vitro and in vivo. Int J Gynecol Cancer. 2014; 24:635-642. 